\title{
Coil Closure of Isolated Aortopulmonary Collaterals in an Infant Presenting with Recurrent Lower Respiratory Tract Infection
}

\author{
Hamid Amoozgar ${ }^{1}$, Mohammad Reza Edraki ${ }^{1}$, Nima Mehdizadegan ${ }^{1}$, Amir Naghshzan ${ }^{1,}{ }^{*}$ and Hamid \\ Arabi $^{2}$ \\ ${ }^{1}$ Neonatal Research Center and Cardiovascular Research Center, Shiraz University of Medical Sciences, Shiraz, Iran \\ ${ }^{2}$ Department of Pediatric, Nemazee Hospital, Shiraz University of Medical Sciences, Shiraz, Iran \\ "Corresponding author: Amir Naghshzan, Pediatric Cardiology Ward, Nemazee Hospital, Shiraz University of Medical Sciences, Shiraz, Iran. Tel: +98-9171133732, E-mail: \\ amirnaghshzan@gmail.com
}

Received 2017 November 21; Revised 2018 February 08; Accepted 2018 February 18.

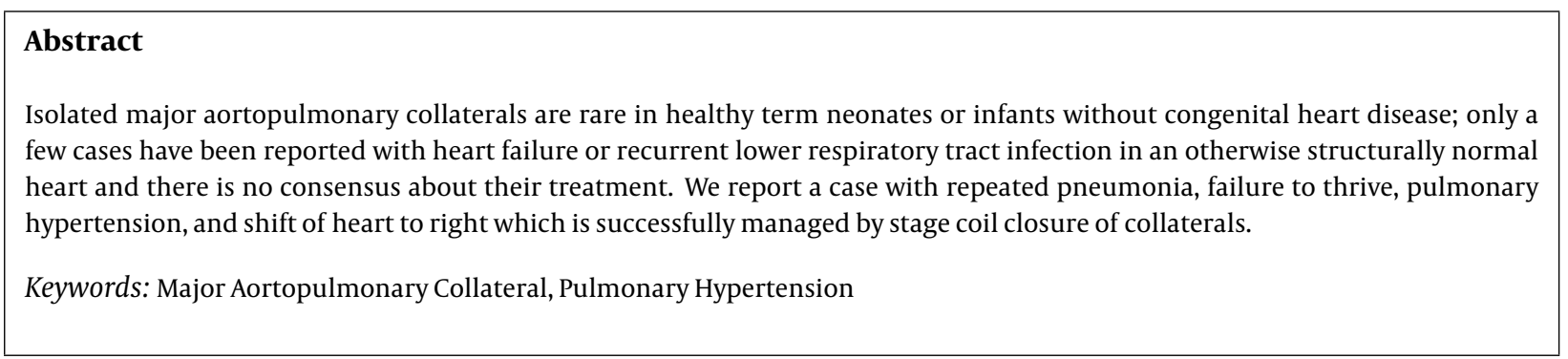

\section{Introduction}

In complex cyanotic congenital heart diseases such as tetralogy of Fallot (TOF) and pulmonary atresia with ventricular septal defect (VSD), major aortopulmonary collaterals (MAPCAs) are the lifesaving pathways for blood supply of the lungs. MAPCAs are incidental findings in neonates, especially in premature neonates with chronic bronchopulmonary disorder (1). Isolated MAPCAs are rare in healthy term neonates or infants without congenital heart disease, and only a few cases have been reported with heart failure or recurrent lower respiratory tract infection in an otherwise structurally normal heart $(1,2)$. These clinical presentations are sometimes related to increased pulmonary blood flow and symptoms may not be controlled by conservative or medical treatment alone.

In complex cyanotic congenital heart disease, MAPCA closure should be done via percutaneous catheter approach, immediately before total surgical correction. In the past, surgery preferred the approach to ligate abnormal vessel communications, but over the past several years, the catheter approach has replaced surgical ligation (3). In some cases, trials of transcatheter occlusion of MAPCAs fails due to the abnormal tortuous course of vessels, hence the patient should be referred for surgery (4).

\section{Case Presentation}

A 6-month-old girl, weighing $5 \mathrm{~kg}$, was followed by a pediatrician due to poor weight gain, recurrent pneumonia and abnormal heart sound in the right hemi thorax. She was delivered at term without any prenatal complications weighing $3 \mathrm{~kg}$.

She had mild central cyanosis with oxygen saturation of $85 \%$, faint pan-systolic murmur on the left sternal border and failure to thrive.

Radiography of the chest showed a shift of heart and mediastinum to the right side and pulmonary congestion (Figure 1).

Echocardiogram showed shift of heart to the right side, very small pinhole ventricular septal defect, medium size atrial septal defect $(5 \mathrm{~mm})$, and trivial tricuspid regurgitation.

During the first hospitalization treatment of pneumonia with appropriate antibiotics and follow up by a pediatric cardiologist and pediatric pulmonologist was done. Spiral chest computerized tomography scan with contrast (Figures 2 and 3) showed a shift of heart, some area of ground glass density in the right lung, multiple tortuous vessels in the left hemi thorax with origins from descending aorta, and drainage to the left pulmonary vein.

After stabilization, first cardiac catheterization was done. Innominate vein angiogram showed rudimentary left superior vena cava. Right pulmonary artery angiogram 


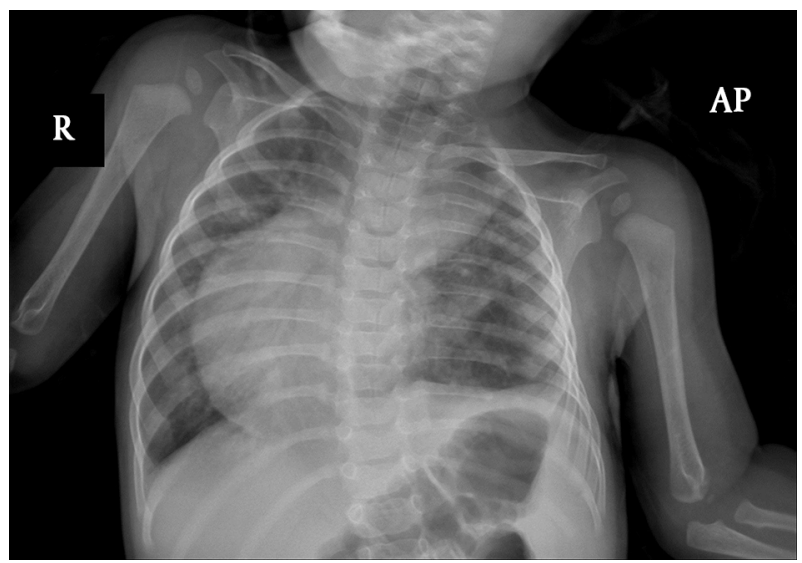

Figure 1. The chest radiography showed shift of heart and mediastinum to the right side and pulmonary congestion.

showed drainage of the right pulmonary veins to the left atrium in levo-phase. Left pulmonary artery angiogram showed small left pulmonary artery mainly supplied the left lower lung and faint flow to the left upper lobe. In continuation, left pulmonary veins drained to the left atrium. Pulmonary pressure measurement revealed mean PA pressure of $26 \mathrm{mmHg}$ and saturation at 76.5\%. Descending aortogram in antro-posterior view showed multiple large collaterals to the left lung and shift of the heart to the right side of the thorax (Figure 4).

All documents were presented to a committee consisting of a pediatric cardiologist, pediatric cardiac surgeon, and pediatric surgeon. The patient was scheduled for percutaneous MAPCAs closure in Namazi hospital, affiliated with Shiraz University of Medical Sciences, Shiraz, Iran.

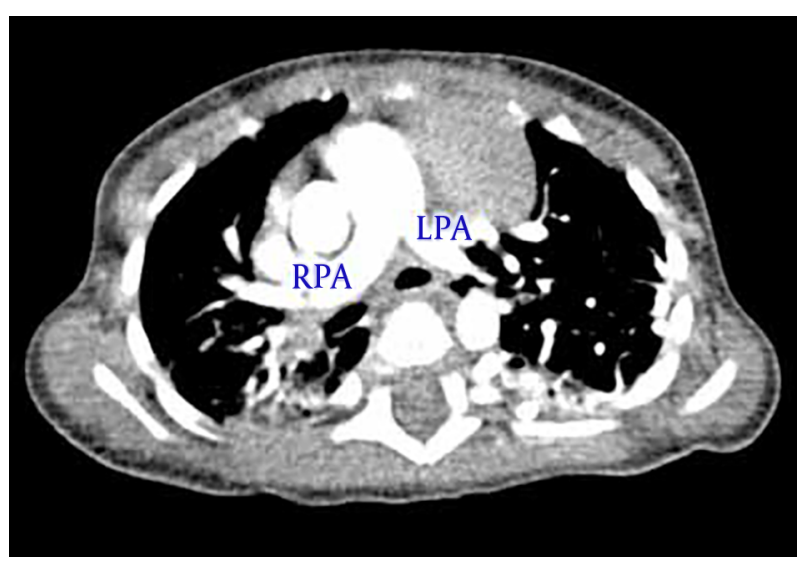

$\overline{\text { Figure 2. Spiral chest CT scan with contrast showed normal anatomy of pulmonary }}$ arteries.

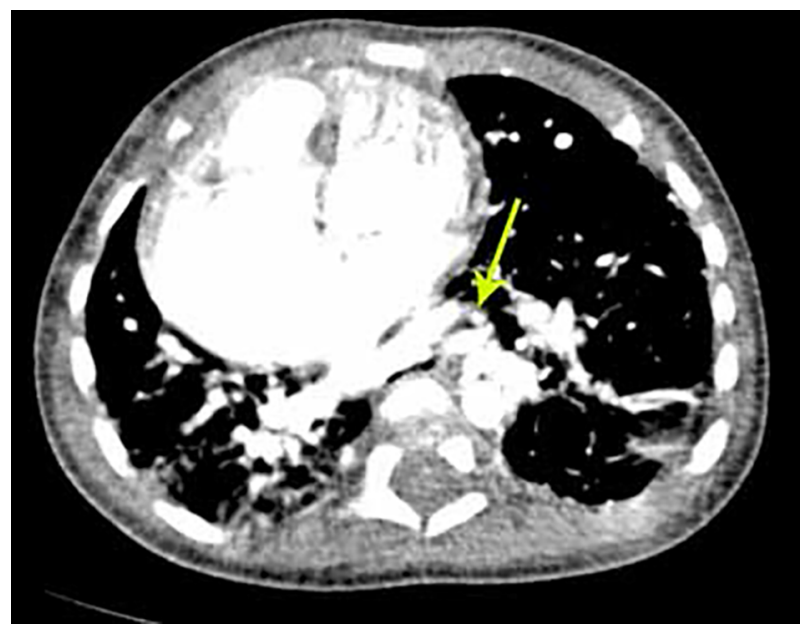

Figure 3. Spiral chest computerized tomography scan with contrast showed abnormal arterial connection of aorta to left lung.

The first collateral closure was performed by two MReye Emobilization Coil IMWCE-35-35 for collateral to mid part of the left lung. The second collateral closure was done by one MReye Emobilization Coil IMWCE-35-35 for collateral to the upper part of the left lung. After right axillary artery access, the 3rd collateral closure was performed by one MReye emobilization Coil IMWCE-35-35 for collateral to mid portion of the lower part of the left lung, and the last collateral closure was performed by one MReye emobiliza-

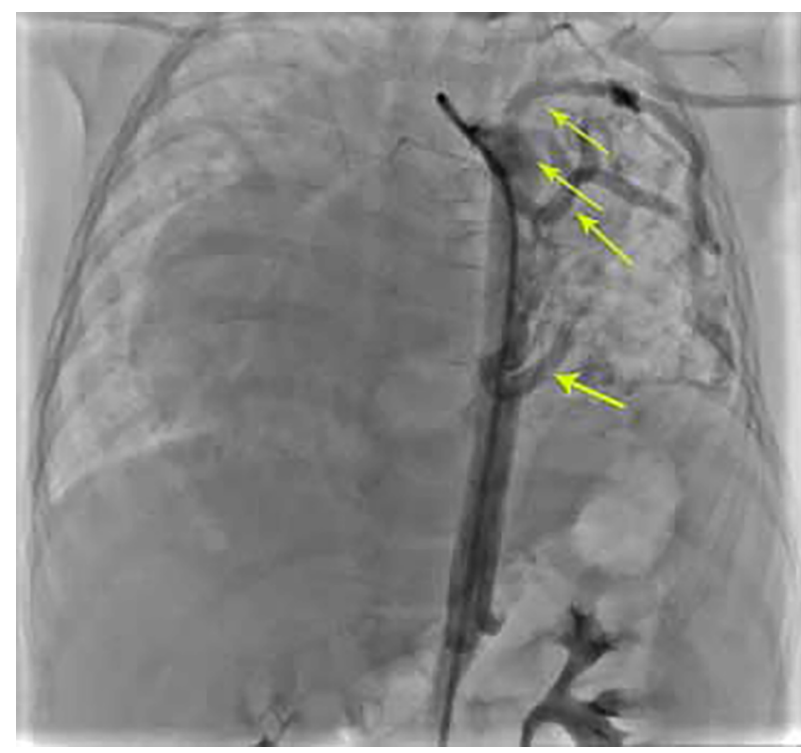

Figure 4. Descending aortogram in antro-posterior view showed multiple large collaterals to the left lung and shift of the heart to the right side of thorax. 


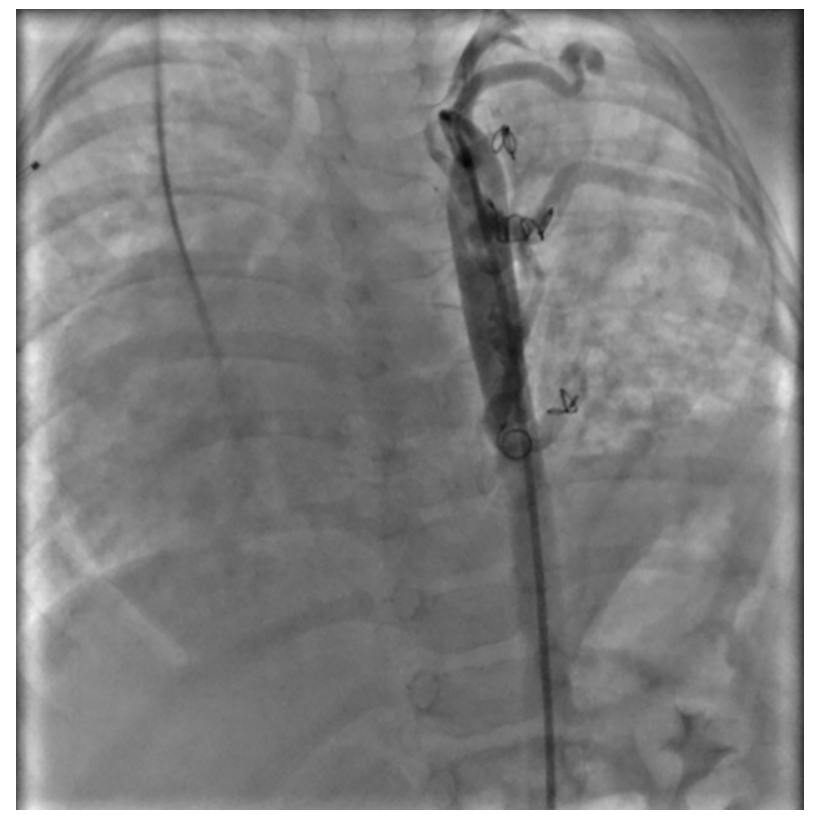

Figure 5. Post procedure aortogram in AP view showed coils were in appropriate position with mild and acceptable residual through the devices.

tion Coil IMWCE-35-35 for collateral to lower portion of the lower part of the left lung.

Post-procedure aortogram in AP view showed that coils were in appropriate position with mild and acceptable residual flow through the devices (Figure 5).

After this procedure the patient was stable for 4 months and then she was scheduled for diagnostic cardiac catheterization to evaluate other collaterals and pulmonary pressure. Echocardiography showed pulmonary hypertension improvement.

Cardiac catheterization showed no pulmonary hypertension (mean PA pressure $24 \mathrm{mmHg}$ ). Aortogram showed residual in one collateral that was closed by the coil in the previous angiography with multiple MAPCAs to the left lung (Figure 6).

The collateral closure was done by PFM coil $7 \times 6 \mathrm{~mm}$ for the left upper lobe and another one by PFM coil $5 \times 4$ $\mathrm{mm}$ for the left middle part of the lung. Post-procedure angiogram showed new devices in appropriate place with minimal leakage.

And now, about 1 year after the first intervention, aortogram in AP view shows no new collateral formation and no residual flow through the previous collaterals except for a minimal residual flow from the small collateral to lower part of left lung (Figure 7) and PA pressure measurement revealed mean pulmonary artery pressure of 23 $\mathrm{mmHg}$.

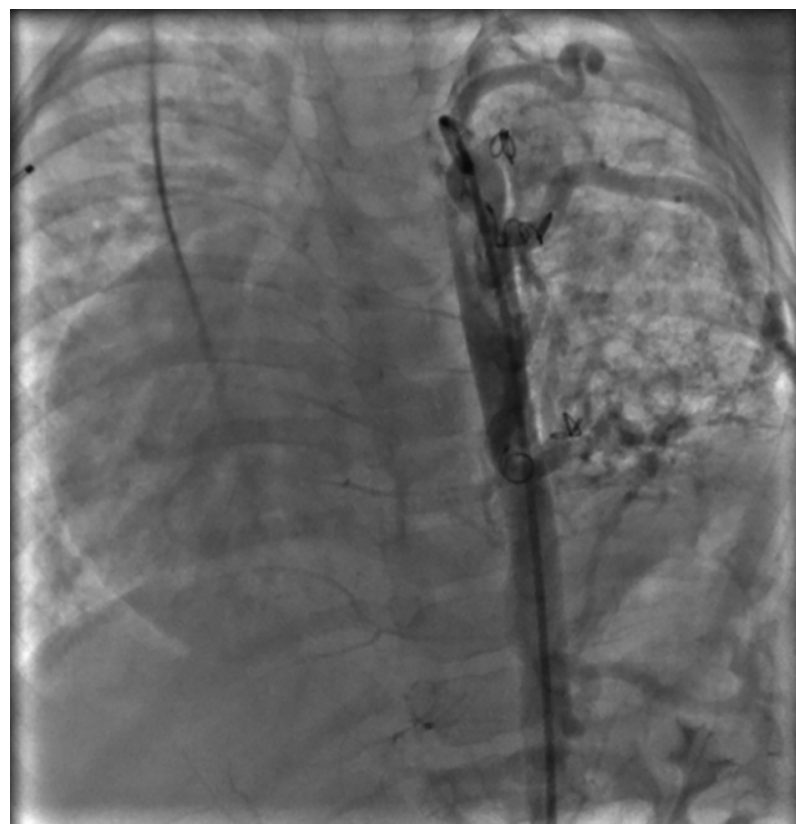

Figure 6. Aortogram in AP view before the second intervention showed residual flow through the previous collateral and also two MAPCAs to LT lung.

\section{Discussion}

The cause of persistent collateral vessels is not clear. However, in an animal study these large vessels connect the

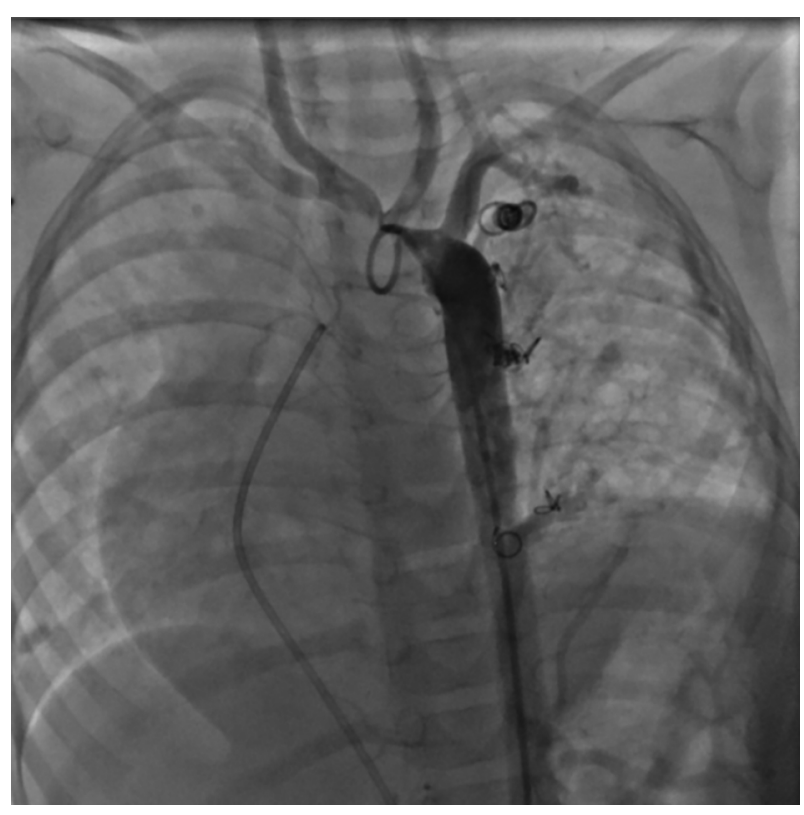

Figure 7. Follow up aortogram after one year with no new collateral formation and only minimal residual from small collateral to lower part of left lung. 
pulmonary plexus with the dorsal aorta early in fetal life (1, 5).

There are some hypotheses for the persistence of these vessels in human but are not confirmed yet. These studies suggest other external factors for persistency of abnormally dilated arteries that connect the bronchial arteries to the pulmonary circulation such as hypoxia or inflammation in cases of bronchopulmonary dysplasia and other cases of neonatal pulmonary injury and trauma $(1,6,7)$.

In these hypotheses hypoxia and inflammation are a stimulus for the production of angiogenesis factors and also blocker of inhibitory factors of angiogenesis. However, in our case and also the two previous $(1,2)$ case reports no disposing factors were detected.

The clinical presentation of patients in previous reports with isolated MAPCA were right-sided heart failure (1, 8 ) and repeated pneumonia (2). Our case presentation is a repeated complicated pneumonia that was secondary to overflow lung due to multiple MAPCAs. Osman Guvenç et al. reported an infant who underwent patent ductus arteriosus and MAPCA occlusion in the same session, with no cyanotic congenital heart disease and recurrent pneumonia (9).

Previous studies in children with congenital heart disease and MAPCA showed that percutaneous embolization has become the treatment of choice for occlusion of these anomalous vessels (3, 10-13). A number of different embolization devices are available including coils, plugs, microspheres, glue, and occlusion balloons (10). Over time, detachable coils have become the most popular devices used in children. However, multiple coils are required to achieve complete occlusion of large vessels. The most frequent complication in this method is residual flow $(3,13)$, using vascular plug can reduce occluded residual.

In a report by Hijazi, coils are limited to small vessels and their usage can be hazardous in case of large and tubular vessels due to the risk of migration and inability to control their delivery (3). According to these factors, in our patient MAPCA closure was done with multiple coils successfully.

After final procedure, the patient became stable with improvement in pneumonia and pulmonary hypertension.

\section{References}

1. Padhi SS, Bakshi KD, Shastri RK. Multiple coil closure of isolated aortopulmonary collateral. Ann Pediatr Cardiol. 2010;3(1):65-7. doi 10.4103/0974-2069.64357. [PubMed: 20814478]. [PubMed Central: PMC2921520].

2. Patra S, Srinivas SK, Agrawal N, Jayaranganath M. Isolated major aortopulmonary collateral artery in an infant presenting with recurrent lower respiratory tract infection. BMJ Case Rep. 2013;2013. doi: 10.1136/bcr-2013-200421. [PubMed: 24334468]. [PubMed Central: PMC3863037].

3. Hijazi ZM. New device for percutaneous closure of aortopulmonary collaterals. Catheter Cardiovasc Interv. 2004;63(4):482-5. doi: 10.1002/ccd.20228. [PubMed: 15558770].

4. Karara K, Mady K. A rare cause of machinery murmur. Aorta to left lower pulmonary vein fistula: Is it an isolated major aortopulmonary collateral artery? JESCTS. 2016;24(3):232-7.

5. de Ruiter MC, Gittenberger-de Groot AC, Rammos S, Poelmann RE. The special status of the pulmonary arch artery in the branchial arch system of the rat. Anat Embryol (Berl). 1989;179(4):319-25. [PubMed: 2735526].

6. Botenga AS. The significance of broncho-pulmonary anastomoses in pulmonary anomalies: a selective angiographic study. Radiol Clin Biol. 1969;38(5):309-28. [PubMed: 5371133].

7. Ascher DP, Rosen P, Null DM, de Lemos RA, Wheller JJ. Systemic to pulmonary collaterals mimicking patent ductus arteriosus in neonates with prolonged ventilatory courses. J Pediatr. 1985;107(2):282-4. [PubMed: 4020557].

8. Tinmaswala MA, Saple PP, Gupta A, N P, N A, Amin K. Isolated major aortopulmonary collateral artery causing ccf in a newborn, a case report. IJMRHS. 2015;4(2):471-3. doi: 10.5958/2319-5886.2015.00090.9.

9. Guvenc O, Odemis E, Saygi M, Demir IH. Rare combination and transcatheter treatment during single session in an infant: Patent ductus arteriosus and major aortopulmonary collateral artery concordance. Turk Kardiyol Dern Ars. 2016;44(7):606-8. doi: 10.5543/tkda.2016.65712. [PubMed: 27774973].

10. Wiegand G, Sieverding L, Bocksch W, Hofbeck M. Transcatheter closure of abnormal vessels and arteriovenous fistulas with the Amplatzer vascular plug 4 in patients with congenital heart disease. Pediatr Cardiol. 2013;34(7):1668-73. doi: 10.1007/s00246-013-0701-9. [PubMed: 23591802].

11. Brzezinska-Rajszys G. Systemic arteriovenous fistulas. In: Sievert H, Qureshi S, Hijazi ZM, Wilson N, editors. Percutaneous interventions for congenital heart disease. London: CRC Press; 2007. p. 411-8.

12. MacDonald ST, Carminati M, Butera G. Initial experience with the Amplatzer Vascular Plug IV in congenital heart disease: coronary artery fistula and aortopulmonary collateral artery embolization. J Invasive Cardiol. 2011;23(3):120-4. [PubMed: 21364243].

13. Tissot C, da Cruz E, Beghetti M, Aggoun Y. Successful use of a new Amplatzer Vascular plug for percutaneous closure of a large aortopulmonary collateral artery in a pulmonary atresia with ventricular septal defect prior to complete repair. Int JCardiol.2007;116(2):e39-41. doi: 10.1016/j.ijcard.2006.07.142. [PubMed: 17052781]. 